\title{
A new quantitative PCR multiplex assay for rapid analysis of chromosome 17p11.2-12 duplications and deletions leading to HMSN/HNPP
}

\author{
Christian T Thiel ${ }^{1}$, Cornelia Kraus ${ }^{1}$, Anita Rauch ${ }^{1}$, Arif B Ekici ${ }^{1}$, Bernd Rautenstrauss ${ }^{1}$ and \\ André Reis*,1 \\ ${ }^{1}$ Institute of Human Genetics, University of Erlangen-Nuremberg, Schwabachanlage 10, 91054 Erlangen, Germany
}

A 1.4-Mb tandem duplication, including the gene for peripheral myelin protein 22 (PMP22) in chromosome $17 \mathrm{p} 11.2-12$ is responsible for $70 \%$ of the cases of the demyelinating type 1 of CharcotMarie-Tooth disease or hereditary motor and sensory neuropathy I (CMT1A/HMSN I). A reciprocal deletion of this CMT1A region causes the hereditary neuropathy with liability to pressure palsies (HNPP). The CMT1A duplication increases the PMP22 gene dosage from two to three, the HNPP deletion reduces the gene dosage from two to one. Currently, routine diagnosis of HMSN/HNPP patients is mainly performed with polymorphic markers in-between the repetitive elements flanking the CMT1A region. These show quantitative and/or qualitative changes in case of a CMT1A duplication and a homozygous allele pattern in case of HNPP deletion. In HNPP patients the deletion is usually confirmed by fluorescence in situ hybridisation (FISH). We now developed a reliable, single tube real-time quantitative PCR assay for rapid determination of PMP22 gene dosage directly. This method involves a multiplex reaction using FAM labelled Taqman-probe with TAMRA quencher derived from PMP22 exon 3 and a VIC labelled probe with non-fluorescent quencher from exon 12 of the albumin gene as internal reference. Copy number of the PMP22 gene was determined by the comparative threshold cycle method $(\Delta \Delta \mathrm{Ct})$. Each sample was run in quadruplicate and analysed at two different threshold levels. The level giving the smallest standard deviation was scored. We evaluated this method through the retrospective analysis of 252 HMSN patients with known genotype and could confirm the previous findings in $99 \%$ of cases. Two patients were wrongly diagnosed with microsatellite analysis while quantitative real-time PCR identified the correct genotype, as confirmed by FISH. Thus, this method shows superior sensitivity to microsatellite analysis and has the additional advantage of being a fast and uniform assay for quantitative analysis of both CMT1A and HNPP.

European Journal of Human Genetics (2002) 11, 170 -178. doi:10.1038/sj.ejhg.5200920

Keywords: HMSN; CMT1A; HNPP; real-time PCR; neuropathy; quantitative assay

\section{Introduction}

Duplication and deletion of the $1.4-\mathrm{Mb}$ region in $17 \mathrm{p} 11.2-$ 12 that is delimited by two 24-kb low copy number repeats (CMT1A-REPs) represent frequent genomic rearrangements

\footnotetext{
${ }^{*}$ Correspondence: Prof. Dr. med. A. Reis, Schwabachanlage 10, 91054 Erlangen, Germany. Tel: +49-9131-8522318; Fax: +49-9131-209297;

E-mail: reis@humgenet.uni-erlangen.de

Received 26 July 2002; revised 1 October 2002; accepted 9 October 2002
}

resulting in two common inherited peripheral neuropathies, Charcot-Marie-Tooth disease (CMT1A) and hereditary neuropathy with liability to pressure palsy (HNPP). ${ }^{1-5}$ CMT1A is the most frequent inherited peripheral neuropathy, with an estimated prevalence of $\sim 1$ in $2500 .^{6}$ About $70 \%$ of the cases of the demyelinating form of Charcot-Marie-Tooth disease (CMT1A/HMSN I) are caused by the autosomal dominantly inherited $1.4-\mathrm{Mb}$ submicroscopic duplication, encompassing the peripheral 
myelin protein 22 (PMP22) gene. $^{7-10}$ In contrast, the submicroscopic deletion of the same region results in hereditary neuropathy with liability to pressure palsy, a distinct form of inherited peripheral neuropathy with episodic and milder manifestation. ${ }^{2-4}$ The CMT1A duplication and the HNPP deletion are caused by unequal crossing over and reciprocal recombination between the flanking 24$\mathrm{kb}$ homologous sequences (CMT1A-REPs). ${ }^{11}$ The gene for the peripheral myelin protein 22, a component of the peripheral nervous system myelin, is contained within the $1.4 \mathrm{Mb}$ region and its gene copy variation is thought to be the underlying cause of disease..$^{7,8,12-14}$

Molecular diagnosis of CMT1A and HNPP involves the detection of gene dosage differences caused by duplication or deletion of the $1.4-\mathrm{Mb}$ region. In the past Southern blots and pulsed-field gel electrophoresis were widely used methods. ${ }^{1,15-18}$ More recently fluorescence in situ hybridisation (FISH) was mainly used for the detection of HNPP by loss of one signal. ${ }^{19-21}$ Several methods with direct allele-specific amplification of the junction fragments by PCR (repeat PCR) have also been reported. ${ }^{22-24}$ Routine analysis for CMT1A and HNPP is mainly based on genotype analysis, involving detection of dosage differences or gain of heterozygosity for microsatelitte alleles or other polymorphic markers within the $1.4-\mathrm{Mb}$ region flanking the PMP22 gene. $^{25-28}$ Although this method is widely used and powerful, it is an indirect method and depends on the informativeness of the markers.

Real-time quantitative PCR has been used previously to detect relative gene expression and amplification, ${ }^{29,30}$ quantitation of bacterial or viral pathogens, ${ }^{31}$ as well as gene dose detection. The possibility of direct measurement of the PCR product accumulated after each cycle, without any intermediate steps, ensures the high specificity of real-time PCR assays. Application of the method for detection of haploidy or triploidy in genetic diseases is a particularly demanding task, because alteration by a factor of 0.5 or 1.5 need to be diagnosed unambiguously. ${ }^{32}$ Two real-time PCR methods for detection of gene dosage of the $1.4-\mathrm{Mb}$ region have been reported. Both use relative quantitative methods to calculate the gene copy number. Wilke et al. ${ }^{32}$ used fluorogenic probes to amplify a region of the exon 4 of the PMP22 gene and the factor VII gene exon 3 as reference. Reactions for the PMP22 test locus and factor VII reference locus were prepared in parallel and amplified in separate runs. The standard curve method was used to determine the relative gene copy number of PMP22. Although the results showed in principle that real-time PCR can be used for precise measurement of gene copy number, the method involves amplification of the test locus and the reference locus in separated reactions. In addition, the use of the standard curve method limits the number of samples measured in one pass. A second approach was chosen by Aarskog and Vedeler. ${ }^{33}$ They used the $\Delta \Delta \mathrm{Ct}$ method to determine the PMP22 gene copy number of 64 samples. This method involved the separate amplification of PMP22 and an internal reference, human serum albumin, using fluorogenic TaqMan probes.

Neither of these two methods has found its way into routine diagnostic analysis of HMSN/HNPP despite the obvious theoretical advantages of these methods. We therefore developed a novel uniform assay for rapid detection of duplication and deletion of the PMP22 gene causing HMSN/HNPP. We established a multiplex TaqMan Assay using fluorogenic probes and specific primers for PMP22 exon 3 and human serum albumin exon 12. Calculation of the gene copy number was performed using the comparative $\mathrm{Ct}$ method $(\Delta \Delta \mathrm{Ct})$. The method was evaluated by means of 252 samples suspected of HMSN/HNPP previously investigated using polymorphic markers, FISH and Southern blot.

\section{Materials and methods \\ Patients}

We investigated 252 patients previously referred for molecular studies of HMSN/HNPP. Analysis of polymorphic markers derived from chromosome 17p11.2-12 was performed for all patients. ${ }^{25-28}$ Some had additional two colour, interphase FISH analysis done using PAC $150 \mathrm{M} 2$ and $178 \mathrm{~F} 10^{19-21}$, as well as Southern blot studies as described previously ${ }^{1,15-18}$ (Table 1). These results indicated 19 HNPP patients, of whom four were confirmed by FISH and three by EcoRI/SacI Southern blot (probe pLR7.8). Forty-six patients showed markers with three alleles or a difference in gene dosage diagnosed as CMT1. In 187 cases a normal gene copy number was diagnosed. For validation of the quantitative real-time PCR assay all samples were tested blind to the previous diagnosis. In addition, two control samples of known genotype were used as calibrator sample.

\section{DNA extraction}

Genomic DNA was extracted from peripheral blood samples of the 252 patients by the salting-out method. ${ }^{34}$ We used the OD260/280 method on a photometer to determine the appropriate DNA concentration. DNA was diluted in HPLC- $\mathrm{H}_{2} \mathrm{O}$ to a concentration of app. $50 \mathrm{ng} / \mu \mathrm{l}$ and stored at $4^{\circ} \mathrm{C}$.

\section{Primer and probes}

TaqMan primers and probes were newly designed according to the manufacturers recommendations with the Primer Express 2.0 Software from Genebank sequences No. XM_08283 and No. NM_000477 and purchased from Applied Biosystems (Weiterstadt). Primers for PMP22 Exon 3 and albumin Exon 12 were chosen with nearly identical predicted melting temperature and a GC-content of 45 to $57 \%$. All four primers were confirmed not to form primer dimer. PMP22 exon 3 forward primer: 5'-GCCACCATGATCCTGTCGAT-3'; PMP22 exon 3 reverse primer: 5'CCCTTGGTGAGGGTGAAGAGT-3'; Human serum albumin 
Table 1 Microsatellite analysis of patients suspected of HMSN/HNPP

\begin{tabular}{lllll}
\hline Diagnosis & Polymorphic markers & Confirmed & FISH & Not confirmed \\
\hline Normal & 187 & 12 & 1 & Southern blot \\
Duplication & 46 & n.d. & not confirmed & n.d. \\
Deletion & 19 & 4 & n.d. & n.d. \\
\hline
\end{tabular}

Results of 252 samplers suspected of HMSN/HNPP referred for molecular studies. Analysis by polymorphic markers suggested 187 unaffected (diploid) samples. Forty-six samples showed at least three alleles in one marker and one significant dosage difference in as least another marker or a dosage difference in at least two markers. Diagnosis CMT1A was given for these samples. Nineteen samples indicated a gene deletion because of only one allele in at least six markers. Four of whom were confirmed by FISH analysis and three by Southern blot analysis. n.d.=not done.

exon 12 forward primer: 5'-AATGCTGCACAGAATCCTTGGT-3'; Human serum albumin exon 12 reverse primer: 5'TCATCGACTTCCAGAGCTGAAA-3'. The PMP22 probe was designed as a standard probe with a fluorophore 5'FAM as reporter and 3'TAMRA as quencher. For the multiplex assay the human serum albumin probe contained the fluorophore 5'VIC and a non-fluorescent Quencher (ABI). PMP22 exon 3 TaqMan probe: 5'-TTCAGCATTCTGTCTCTGTTCCTGTTCTTCTG-3'; human serum albumin exon 12 TaqMan probe: 5'-ACAGGCGACCATGC-3'. Optimal concentration of primers, probes and template resulting in the highest intensity and specificity of each reporter fluorescent signal were determined on separate multiplexed optimising pre-runs.

\section{TaqMan reaction}

The multiplex assay for each sample was performed in quadruplicate in one 384-well optical plate (Applied Biosystems) with a final volume of $15 \mu \mathrm{l}$ each. All reactions of each plate were prepared from a single PCR master mix consisting of: $2 \times$ TaqMan Mastermix (Eurogentec, Belgium), $1200 \mathrm{nM}$ human serum albumin forward primer, $1200 \mathrm{nM}$ human serum albumin reverse primer, $100 \mathrm{nM}$ human serum albumin probe, $300 \mathrm{nM}$ PMP22 forward primer, $300 \mathrm{nM}$ PMP22 reverse primer and $100 \mathrm{nM}$ PMP22 probe. A total of 50250 ng DNA was dispensed with a Hydra-System (Hydra96, Robbins Scientific, Sunnyvale, USA) in each of the four sample wells for quadruplicate reactions. Various batches of reagents were used in the course of experiments without affecting the results. Optimising preruns revealed the DNA concentration not to be an important factor in the quantification of the PCR reaction. Optimum amplification was detected in the range of 50-250 ng DNA per reaction well, while lower concentrations showed unstable amplification. After distribution of the PCR master mix to each well the reaction was mixed thoroughly with the Hydra-System and the 384-well sample tray was centrifuged briefly at 4000 rpm for $3 \mathrm{~min}$. The sample tray was covered with an adhesive cover and the reaction carried out in an ABI Prism 7900HT Sequence Detection System (Applied Biosystems). Thermal cycling conditions included a prerun of $2 \mathrm{~min}$ at $50^{\circ} \mathrm{C}$ and $10 \mathrm{~min}$ at $95^{\circ} \mathrm{C}$. Cycle conditions were 45 cycles at $95^{\circ} \mathrm{C}$ for $15 \mathrm{sec}$ and $60^{\circ} \mathrm{C}$ for $1 \mathrm{~min}$ according to the TaqMan Universal PCR Protocol (ABI).

Two known control samples (diploid samples) were analysed on each reaction tray for the purpose of calibration as required in the $\Delta \Delta$ Ct-Method. In addition, each plate run was monitored with four control samples, two with typical HNPP deletion (haploid samples) and two with typical CMT1A duplication (triploid samples). Amplification plots were reviewed after performing the reaction and the baseline settings were adjusted when applicable. Reaction wells with obvious PCR reaction failure were excluded from further data evaluation.

\section{Data analysis}

Data evaluation was carried out using the ABI Prism sequence detection system and Microsoft Excel. Amplicons were run in quadruplicate in a one tube assay for the quantification of PMP22 genes to the internal control gene, albumin. The threshold cycle number (Ct) was determined for all PCR reactions. The Ct parameter represents the cycle number $(\Delta \mathrm{Rn})$ at which the amplification plot, representing the fluorescence emission of the reporter dye, passed a fixed threshold. The threshold was automatically set at 10 standard deviations (SD) above the mean baseline emission. In most cases the threshold was manually adjusted within the logarithmic curve, above background level and below the plateau phase. Data analysis was performed only for samples with at least three amplifications (triplicates).

The same threshold and baseline were set for all samples including the calibrator samples. Using the $\Delta \Delta$ Ct-Method, the starting copy number of the samples was determined: $\Delta \mathrm{Ct}$ represents the mean $\mathrm{Ct}$ value of each sample and was calculated for PMP22 and albumin. The starting copy number of the unknown samples was determined relative to the known copy number of the calibrator sample using the following formula: $\Delta \Delta \mathrm{Ct}=[\Delta \mathrm{Ct}$ albumin (calibrator sample) $-\Delta$ Ct PMP22 (calibrator sample) $]-[\Delta \mathrm{Ct}$ albumin (unknown sample)- $\Delta$ Ct PMP22 (unknown sample)]. ${ }^{33}$ The relative gene copy number was calculated by the expression $2^{-(\Delta \Delta \mathrm{Ct}+/-\mathrm{s})}$, where $\mathrm{s}$ represents the difference of the mean standard deviations SD of the Ct values of PMP22 and albumin. To minimise the variation of the relative gene copy 
number measured in the four amplifications from each sample we performed the analysis at two different thresholds within the logarithmic curve. The standard deviation was calculated for each sample at each threshold and the $\Delta \Delta \mathrm{Ct}$ ratio $\left(2^{-(\Delta \Delta \mathrm{Ct})}\right)$ of that with the smallest standard deviation was scored. Using this calculation a $\Delta \Delta \mathrm{Ct}$ ratio of about 1 for a normal sample, about 0.5 for a sample with a gene deletion (HNPP) and about 1.5 for a sample with gene duplication (CMT1A) is expected.

\section{Results}

\section{Assay development}

Initially we tested two combinations of primers and probes for the albumin locus. For this study we used the albumin primers and probes resulting in the highest amplification quality. The initially designed primers and probes for the PMP22 locus did not need further optimisation. Separate and multiplex pre-runs varying the concentrations of PMP22 and albumin primers and probes identified the conditions for the parallel amplification with the same plateau phase and end-point of amplification, respectively. The amplification plot of known diploid samples revealed a slightly slower amplification of the human serum albumin segment compared to the PMP22 segment, resulting in a shift of the amplification curve to the right. Therefore, primer concentration of the albumin gene had to be increased fourfold compared to that of PMP22. Since the PCR conditions were identical we combined the PCRs in a multiplex single tube assay. After assay optimisation, no difference in the amplification curve for PMP22 and albumin was noticed between single and multiplex PCR.

Reliability of the PCR was very good. Analysis of 252 samples in quadruplicate resulted in 1008 multiplex PCR assays. A total of 14 samples had to be repeated because of laboratory error resulting in insufficient amplification. Amplification failures in one of the quadruplicates occurred in 15 samples (6\%), while all had at least three scorable amplifications. The overall PCR efficiency of the $15 \mu \mathrm{l}$ assays was $98.5 \%$.

Performing each sample in quadruplicate runs showed almost complete overlap in the exponential and plateau phases of parallel amplification plots. In samples confirmed of a gene copy number difference, duplication as well as deletion, the $\Delta \mathrm{Ct}$ value showed a divergence compared to the $\Delta \mathrm{Ct}$ value of the defined normal sample. A typical example of amplification plots is given in Figure 1. Thus the PMP22 Ct value, when estimated by the comparative Ct method, showed an increase of around 0.5 compared to the known normal samples in a deletion, whereas, in samples positive for a duplication the $\mathrm{Ct}$ value showed a decrease of about 0.5 .

\section{Data analysis}

Minor deviations in the PCR amplification and the fluorescence detection within the logarithmic phase can lead to significant variation in the $\Delta \mathrm{Ct}$ ratio. This may lead to misinterpretation of the gene copy number, as deletion or duplication vary only by a factor of 0.5 and 1.5 , respectively. Therefore, setting the optimal threshold is an important prerequisite to determine the correct gene copy number (Figure 1). To avoid setting individual thresholds for each sample and the associated potential bias, we determined two thresholds semi-manually within the logarithmic phase for all samples. Calculation of the standard deviation of the Ct values of PMP22 and albumin at both thresholds allowed a determination of the threshold with the lowest standard deviation for each sample, the lowest deviation was scored.

Subsequently, we determined the range for detecting deletions and duplications using 40 samples with known genotype (Figure 2). Three groups of samples are clearly distinguishable: Samples with a $\Delta \Delta \mathrm{Ct}$ ratio below 0.6 consistent with a gene copy deletion (HNPP) in agreement with analysis of polymorphic markers, FISH and/or Southern blot; a $\Delta \Delta \mathrm{Ct}$ ratio range of 0.8 to 1.2 confirmed a diploid genotype; samples with a $\Delta \Delta \mathrm{Ct}$ ratio above 1.3 all had a PMP22 duplication (CMT1A) as verified by polymorphic markers with three alleles and/or a dosage difference. The mean values for $\mathrm{e}^{-\Delta \Delta \mathrm{Ct}}( \pm 1 \mathrm{SD})$ for each sample show no overlap in $\Delta \Delta \mathrm{Ct}$ ratios between the three groups of samples (Figure 2). Therefore diagnostic ranges were set for samples confirming a PMP22 gene deletion (HNPP) if the $\Delta \Delta \mathrm{Ct}$ ratio were under 0.6 , a diploid genotype within a $\Delta \Delta$ Ct ratio range of 0.8 to 1.2 and a PMP22 gene duplication (CMT1A) with a $\Delta \Delta \mathrm{Ct}$ ratio range above 1.3. Results outside these ranges were considered inconclusive and the analysis was repeated.

\section{Validation of the assay}

We next proceeded to validate the assay developed using the parameters determined previously. Samples of 252 patients referred for molecular studies, including the 40 used to determine the parameters, were analysed using polymorphic markers as formerly described. ${ }^{27,28}$ Each patient was investigated for at least five microsatellite markers (Table 1). A total of 46 patients showed at least three alleles in one marker and one significant dosage difference in at least another marker, or a dosage difference in at least two independent markers according to the European guidelines for CMT diagnostics (www.EMQN.org), confirming a duplication of chromosome 17p11.2-12 region in these patients. Nineteen samples showed only one allele in all markers, suggesting deletion and therefore HNPP as the likely diagnosis. Of these, four samples were confirmed as deleted by FISH analysis and three by Southern blot analysis. One sample homozygous for all seven markers analysed, and thus suggesting a deletion in the microsatellite analysis, was diagnosed as normal in the FISH analysis. The remaining 187 samples showed a normal gene copy number in the microsatellite analysis. Out of these samples 13 were also confirmed by FISH analysis (Table 1). 


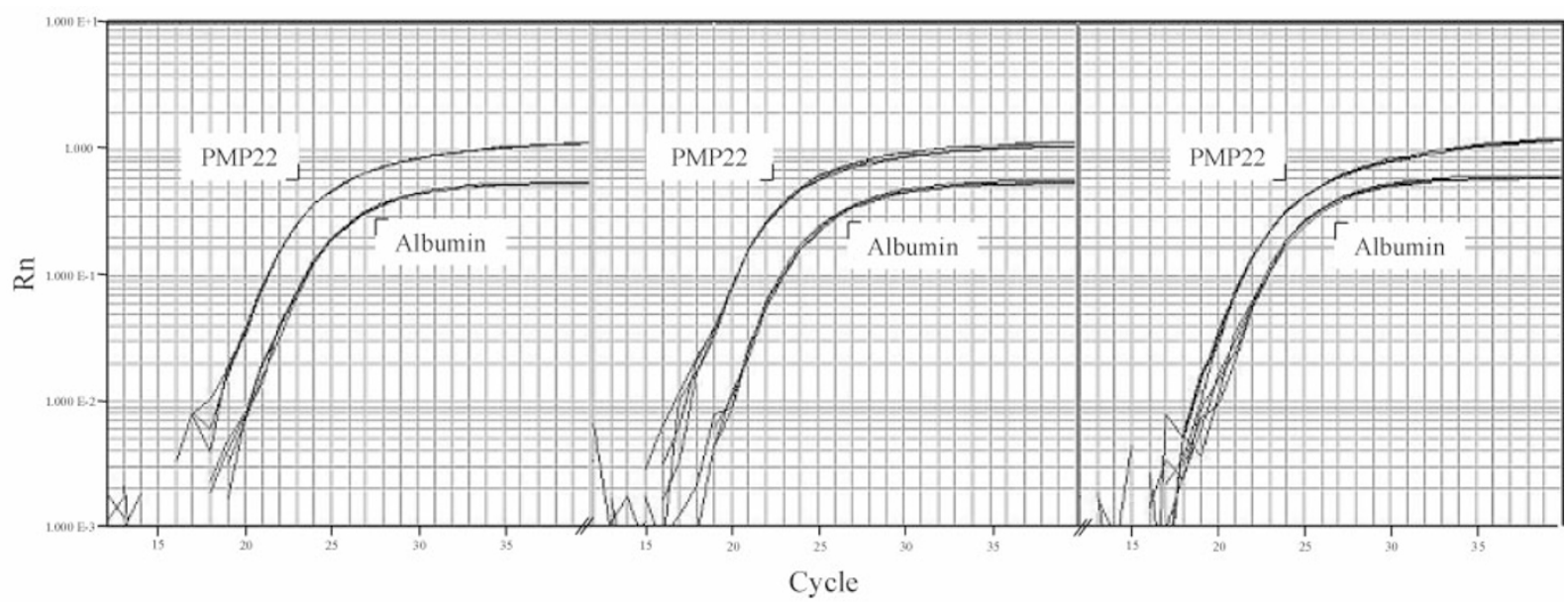

Figure 1 Amplification plot of peripheral myelin protein 22 (PMP22) and albumin in a multiplex single tube assay. The quadruplicate runs show almost complete overlap of parallel amplification plots. The $\Delta \mathrm{Rn}$ value represents the quantity of fluorescent probe degraded and fits an exponential function generating an amplification plot for each well (logarithmic scale of the $y$-axis). Degradation of the probe occurred when the probe was cleaved by $5^{\prime}$ nuclease activity of the Taq DNA polymerase. After sufficient hybridisation probe is cleaved, the intensity of the reporter (FAM for the PMP22 probe, VIC for the albumin probe) fluorescent emission increases above background and crosses the threshold. The cycle number is plotted on the $x$-axis. The baseline was automatically set from 3 to 15 cycles and required no adjustment. We used two different thresholds in order to exclude separation of the amplification line of one of the quadruplicate measurements caused by amplification variation of single wells (eg albumin amplification in Plot C lower threshold). The thresholds were set manually within the logarithmic phase according to the manufacturer's instructions. That with the lowest standard deviation difference of PMP22 and albumin was scored. (A) Normal healthy control; (B) PMP22 duplication suggesting CMT1A: the increase in the starting copy number of the PMP22 gene results in an earlier increase of the reporter fluorescent emission and a shift of the amplification plots of the PMP22 PCR to the left, resulting in an increased $\triangle$ Ct value. (C) PMP22 gene deletion suspecting HNPP: decrease in the starting copy number yields a shift of the amplification plot to the right, decreasing the $\Delta \mathrm{Ct}$ value.

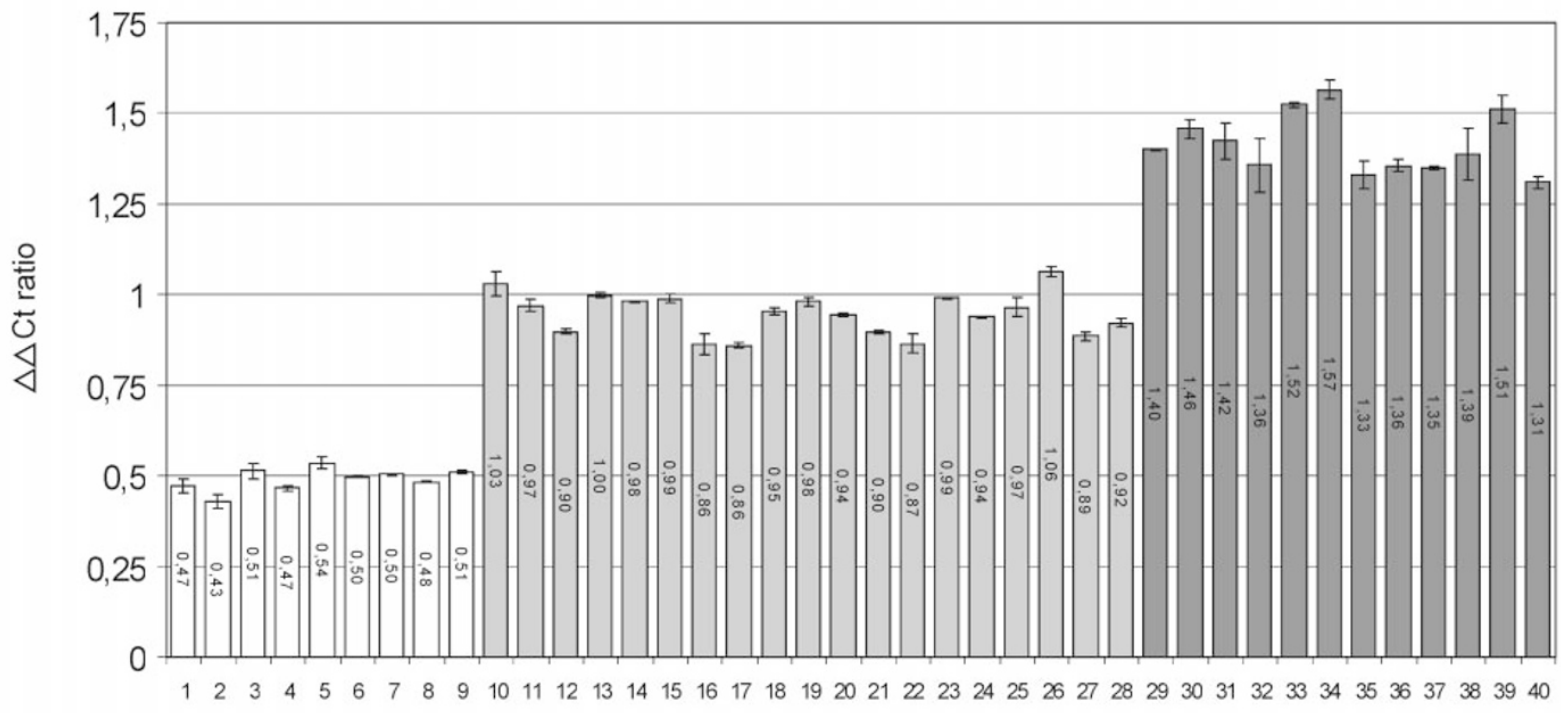

Sample No.

Figure $2 \Delta \Delta$ Ct ratio and standard deviation of 40 samples with known genotype. Nine samples with a deletion (open boxes) show $\Delta \Delta \mathrm{Ct}$ ratios of about 0.5 indicating HNPP, 19 normal samples with $\Delta \Delta \mathrm{Ct}$ ratios of about 1.0 indicating diploid genotype (light grey boxes) and 12 samples with a duplication exhibiting $\Delta \Delta$ Ct ratios above 1.3 indicating CMT1A (dark grey boxes). The real-time PCR confirms the results obtained previously using polymorphic markers, FISH and Southern blot. 
Results for the real-time PCR analysis of the 252 samples are given in Table 2 and Figure 3. Only three of the 252 samples led to a result outside the determined ranges, and were repeated. Careful investigation revealed that the problems experienced with these analyses could be attributed to insufficient mixture of DNA and PCR mix. Subsequent assays with rigorous mixing of reagents permitted a definite diagnosis. Gene copy analysis by real-time PCR showed a dosage difference of about 1.5 of the PMP22 gene in all 46 cases, formerly diagnosed for duplication. A total of 186 of the 187 samples previously diagnosed with normal gene copy number, were also confirmed in the real-time PCR assay. One sample, though, with a normal microsatellite pattern in repeated analyses (four informative markers out of eight analysed) consistently showed a gene dose difference of 1.5 , suggesting duplication of the PMP22 gene. Interphase FISH analysis of this sample confirmed the duplication (data not shown). Eighteen out of nineteen samples were confirmed as HNPP deletion by a gene dose difference of about 0.5 The sample homozygous for all polymorphic markers investigated, but with a normal result in the FISH analysis, was also confirmed to be diploid in real-time PCR.

Overall, the microsatellite analysis was correct in 250 out of 252 (99\%) of the samples investigated. The quantitative

Table 2 Validation of the real-time PCR results

\begin{tabular}{|c|c|c|c|c|c|c|c|}
\hline \multirow[b]{2}{*}{ Diagnosis } & \multirow[b]{2}{*}{ Real-time $P C R$} & \multicolumn{2}{|c|}{ Polymorphic markers } & \multicolumn{2}{|c|}{ FISH } & \multicolumn{2}{|c|}{ Southern blot } \\
\hline & & Confirmed & Not confirmed & Confirmed & Not confirmed & Confirmed & Not confirmed \\
\hline Normal & 187 & 186 & 1 & 13 & 0 & n.d. & n.d. \\
\hline Duplication & 47 & 46 & 1 & 1 & 0 & n.d. & n.d. \\
\hline Deletion & 18 & 18 & 0 & 4 & 0 & 3 & 0 \\
\hline
\end{tabular}

Validation of 252 samples suspected of HMSN/HNPP and previously analysed by polymorphic markers, FISH analysis and Southern blot analysis. A total of 187 samples were suggested as diploid samples, and 186 could be confirmed by analysis of polymorphic markers and 13 by FISH analysis. One sample previously suggested unaffected in the polymorphic marker analysis showed a duplication of the PMP22 gene in repeated runs. Duplication in this sample was confirmed with FISH analysis. All 46 samples previously suggested for a duplication were confirmed in the real-time PCR analysis. Real-time PCR analysis indicated a PMP22 gene deletion in 18 samples, which were confirmed in the analysis of polymorphic markers. Four of the 18 samples were tested with FISH analysis and could be confirmed. One sample homozygous in all seven microsatellites analysed and thus suspected of a deletion of the PMP22 gene, gave normal results in the real-time PCR analysis and was confirmed diploid with FISH. n.d.=not done.

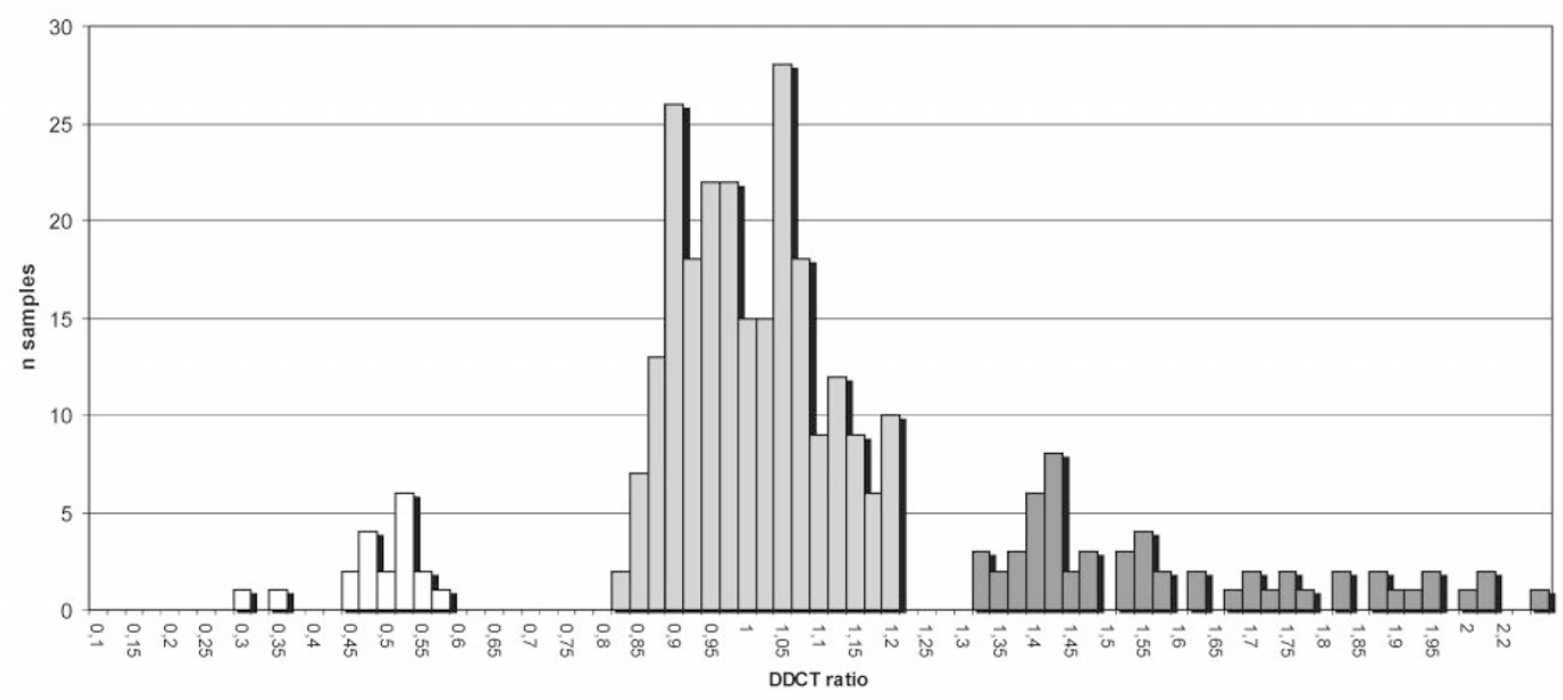

Figure 3 Results of assay evaluation in 252 samples. Mean $\Delta \Delta \mathrm{Ct}$ ratios from 252 patients obtained using the novel quantitative real-time PCR assay as described in the text. Samples confirmed for the diagnosis HNPP (ie deletion) showed a $\Delta \Delta$ Ct ratio range of 0.3 to 0.6 with a mean $\Delta \Delta \mathrm{Ct}$ ratio of 0.48 (open boxes). Samples within a $\Delta \Delta \mathrm{Ct}$ ratio range of 0.8 to 1.2 (mean $\Delta \Delta \mathrm{Ct}$ ratio 0.99 ) indicate a diploid gene copy dose (light grey boxes). A $\Delta \Delta$ Ct ratio range of 1.3 to 2.36 (mean 1.58) indicated a PMP22 gene duplication (dark grey boxes). Diagnostic ranges were clearly separated without any overlap. Validating these findings with the results of the former analyses (polymorphic markers, FISH, Southern blot) confirmed the diagnosis in all 252 samples, thus confirming the high specificity and sensitivity of the method. 
PCR assay described here seems to have made the correct calling in all 252 cases.

\section{Discussion}

Real-time quantitative PCR requires specific equipment, but the method has proven to be fast, precise and reproducible. ${ }^{32,33,35,36}$ The advantage of the real-time PCR is the calculation of the gene copy number based on a $\mathrm{Ct}$ value, rather than using endpoint measurement, where a slight difference in any of the limiting components could have a dramatic effect on the amount of PCR product. The real-time PCR reaction is characterised by an exponential increase in the amount of PCR product in the early cycle, and reaches a plateau phase as the reaction components become limiting. The higher the starting copy number of the target gene, the sooner the increase in fluorescence is detected and a lower $\mathrm{Ct}$ value is reached.

We now established a new multiplex assay for fast and reliable detection of the gene copy number of the PMP22 gene. For an optimum real-time PCR amplification the design of the primers and probes proved to be an important factor. Primers and probes for PMP22 exon 3 and albumin exon 12 (internal standard) were newly designed. Despite use of special software, ie the Primer Express 2.0 program (Applied Biosystems), the most suitable primer pair combination had to be determined empirically. Initially, the PCR plateau phase of the albumin amplification was lower than in the PMP22 amplification, suggesting a more effective PCR of PMP22. ${ }^{33}$ We were able to compensate this through increasing albumin primer and probe concentrations. We thus achieved similar amplification plots for PMP22 and albumin and were able to increase the plateau phase, and accordingly the length of the exponential phase without influencing the $\Delta \Delta \mathrm{Ct}$. This decreased the risk of misinterpreting the results from setting the threshold outside the exponential phase. All assays of this multiplex reaction were carried out in 384-well plates with a reaction volume of $15 \mu \mathrm{l}$. This marked reduction in the reaction volume $(4 \times 15 \mu \mathrm{l})$ as compared to previously published assays $(6 \times 50 \mu \mathrm{l})$ results in greatly reduced reagent costs. A lower reaction volume $(10 \mu \mathrm{l})$ resulted in non-consistent results (data not shown). PCR efficiency is not compromised by this reduction, as we obtained a first pass efficiency of $98.5 \%$ and were able to amplify at least three out of four wells for all samples with no more than two attempts. As expected, our results also emphasize the need for uniform high DNA quality to ensure consistent amplification plots, and thus, a low standard deviation of the quadruplicates. DNA concentration was less critical, and gave good results, in a range of 50-250 ng/sample.

Both previously reported real-time PCR assays to quantify the gene copy number of PMP22 for diagnosis of CMT1A/ HNPP were designed as 2-well assays. ${ }^{32,33}$ In addition, the $\Delta \Delta \mathrm{Ct}$ method described by Aarskog et al. ${ }^{33}$ requires largescale PCR assays $(2 \times 50 \mu \mathrm{l})$ to prevent laboratory errors when using a standard amplified in a different well. The standard curve method from Wilke et al. ${ }^{32}$ requires a large amount of standard samples. Both methods are expensive and limited in throughput due to reagent cost and manual labour involved.

Even when handling all pipetting steps for DNA and reagent mix dispensing with an automatic dispensing device, PCR amplification plots of the same sample may vary. If the difference in gene dosage is as low as in CMT1A/HNPP this may lead to incorrect measurement due to chance fluctuation in the assay, especially if a uniform threshold is used over a series of samples. We, therefore, chose to perform four individual measurements of each sample (quadruplicates) to increase the accuracy. To prevent the risk of misinterpreting the mean $\mathrm{Ct}$ value at a set threshold and in order to be able to set the threshold for a huge amount of samples at the same time, we used two different threshold values within the exponential phases of the amplification of PMP22 and albumin. These two thresholds cross the pairs of amplification plots at different points of the exponential curve. The point where all plot pairs are closest is probably the optimal point for measurement of a given sample and the variance should be lowest. Therefore the Ct value of the threshold with the lowest standard deviation was used for further calculation of the gene copy number.

Having developed the assay we next determined the diagnostic ranges for samples with a PMP22 deletion (HNPP), with a diploid genotype and for samples with a PMP22 duplication (CMT1A). Using 40 samples with known genotype the $\Delta \Delta \mathrm{Ct}$ ratio values of the three groups were clearly distinguishable and showed no overlap, indicating high sensitivity and specificity of this assay (Figure 2). Thus, the definition of ranges for these groups prevents misdiagnosis of samples with inappropriate handling. Diagnostic intervals for our assay were set according to the distribution of the $\Delta \Delta \mathrm{Ct}$ ratio, values to $<0.6$ indicating the PMP22 gene deletion (HNPP), 0.8-1.2 for the diploid genotype, $>1.3$ indicating the PMP22 gene duplication (CMT1A) (Figure 2).

For evaluation of this new one-tube assay we examined 252 samples and compared these results with the former findings of the analysis of microsatellite markers, FISH and Southern blot. All 252 samples had been previously genotyped with microsatellite markers. ${ }^{25-28}$ This method, however, is dependent on the informativity of markers, which are distributed within the $1.4-\mathrm{Mb}$ genomic region. ${ }^{5}$ The FISH analysis is mostly used for detection of the PMP22 gene deletion. ${ }^{20,21}$ It is less well suited for routine diagnosis of PMP22 gene duplications due to the difficulty in distinguishing PMP22 gene duplication from split signals of the probe.

Quantitative PCR was performed blind to initial diagnosis. All 252 samples could be clearly classified into one of the categories based on the previously determined diagnos- 
tic ranges. Three samples showed a $\Delta \Delta \mathrm{Ct}$ ratio outside the ranges and were repeated and permitted a correct diagnosis, indicating liquid handling errors as cause of the inappropriate results. All 48 CMT1A patients with a duplication identified in microsatellite analyses were confirmed with real-time PCR to carry the PMP22 duplication, whilst 14 of 15 patients suggested for HNPP by microsatellite marker analysis were found to have the PMP22 deletion. Patients with diploid genotype according to previous methods numbered 187, of which 186 were confirmed to carry two gene copies in real-time PCR (Table 2).

One sample, a familial case, was informative for 4 out of 8 polymorphic markers investigated. It showed two alleles and no dosage difference in these markers, suggesting an unaffected sample. Real-time PCR showed in repeated analysis a $\Delta \Delta$ Ct ratio $>1.3$ indicating a PMP22 gene duplication. This sample was confirmed to be duplicated by interphase $\mathrm{FISH}$, indicating that this patient might carry an atypical duplication not detected by the microsatellites. In the second conflicting sample suspected for a deletion in microsatellite analysis FISH analysis confirmed the result of the quantitative PCR. Overall, these results suggest that our new one tube real-time PCR assay representing a quantitative and not qualitative analysis is at least equal if not superior to the analysis with polymorphic markers.

In summary, we have developed a uniform, quantitative real-time PCR assay for detection of chromosome 17p11.2-12 duplication and deletion in a single tube. We have shown this assay to be highly sensitive and specific as well as fast and cost effective. In evaluation with 252 previously diagnosed cases we have shown this assay to be equal or even superior to other currently used methods.

\section{References}

1 Lupski JR, de Oca-Luna RM, Slaugenhaupt S et al: DNA duplication associated with Charcot-Marie-Tooth disease type 1A. Cell 1991; 66: 219-232.

2 Chance PF, Alderson MK, Leppig KA et al: DNA deletion associated with hereditary neuropathy with liability to pressure palsies. Cell 1993; 72: 143-151.

3 Chance PF, Pleasure D: Charcot-Marie-Tooth syndrome. Arch Neurol 1993; 50: $1180-1184$

4 Chance PF, Abbas N, Lensch MW et al: Two autosomal dominant neuropathies result from reciprocal DNA duplication/deletion of a region on chromosome 17. Hum Mol Genet 1994; 3: 223-228.

5 Inoue K, Dewar K, Katsanis N et al: The 1.4-Mb CMT1A duplication/HNPP deletion genomic region reveals unique genome architectural features and provides insights into the recent evolution of new genes. Genome Res 2001; 11: $1018-1033$.

6 Skre H: Genetic and clinical aspects of Charcot-Marie-Tooth's disease. Clin Genet 1974; 6: 98-118.

7 Patel PI, Roa BB, Welcher AA et al: The gene for the peripheral myelin protein PMP-22 is a candidate for Charcot-Marie-Tooth disease type 1A. Nat Genet 1992; 1: 159-165.

8 Timmerman V, Nelis E, Van Hul W et al: The peripheral myelin protein gene PMP-22 is contained within the Charcot-MarieTooth disease type 1A duplication. Nat Genet 1992; 1: 171-175.
9 Wise CA, Garcia CA, Davis SN et al: Molecular analyses of unrelated Charcot-Marie-Tooth (CMT) disease patients suggest a high frequency of the CMTIA duplication. Am J Hum Genet 1993; 53: $853-863$.

10 Nelis E, Van Broeckhoven C, De Jonghe P et al: Estimation of the mutation frequencies in Charcot-Marie-Tooth disease type 1 and hereditary neuropathy with liability to pressure palsies: a European collaborative study. Eur J Hum Genet 1996; 4: 25-33.

11 Lupski JR: Charcot-Marie-Tooth disease: lessons in genetic mechanisms. Mol Med 1998; 4: 3-11.

12 Lupski JR, Wise CA, Kuwano A et al: Gene dosage is a mechanism for Charcot-Marie-Tooth disease type 1A. Nat Genet 1992; 1: 29 33.

13 Matsunami N, Smith B, Ballard L et al: Peripheral myelin protein22 gene maps in the duplication in chromosome $17 \mathrm{p} 11.2$ associated with Charcot-Marie-Tooth 1A. Nat Genet 1992; 1: 176179.

14 Valentijn LJ, Bolhuis PA, Zorn I et al: The peripheral myelin gene PMP-22/GAS-3 is duplicated in Charcot-Marie- Tooth disease type 1A. Nat Genet 1992; 1: 166-170.

15 Raeymaekers P, Timmerman V, Nelis E et al: Duplication in chromosome $17 \mathrm{p} 11.2$ in Charcot-Marie-Tooth neuropathy type 1a (CMT 1a). The HMSN Collaborative Research Group. Neuromuscul Disord 1991; 1: 93 -97.

16 MacMillan JC, Harper PS: The Charcot-Marie-Tooth syndrome: perceptions of disability and projected use of DNA diagnostic tests. Clin Genet 1992; 42: 161-163.

17 Raeymaekers P, Timmerman V, Nelis E et al: Estimation of the size of the chromosome 17p11.2 duplication in Charcot- Marie-Tooth neuropathy type 1a (CMT1a). HMSN Collaborative Research Group. J Med Genet 1992; 29: 5-11.

18 Lupski JR: DNA diagnostics for Charcot-Marie-Tooth disease and related inherited neuropathies. Clin Chem 1996; 42: 995 -998.

19 Liehr T, Rautenstrauss B, Grehl H et al: Mosaicism for the CharcotMarie-Tooth disease type $1 \mathrm{~A}$ duplication suggests somatic reversion. Hum Genet 1996; 98: 22-28.

20 Roa BB, Greenberg F, Gunaratne P et al: Duplication of the PMP22 gene in $17 \mathrm{p}$ partial trisomy patients with Charcot-Marie-Tooth type-1 neuropathy. Hum Genet 1996; 97: 642-649.

21 Shaffer LG, Kennedy GM, Spikes AS et al: Diagnosis of CMT1A duplications and HNPP deletions by interphase FISH: implications for testing in the cytogenetics laboratory. Am J Med Genet 1997; 69: 325-331.

22 Haupt A, Schols L, Przuntek H et al: Polymorphisms in the PMP22 gene region (17p11.2-12) are crucial for simplified diagnosis of duplications/deletions. Hum Genet 1997; 99: 688-691.

23 Yamamoto M, Keller MP, Yasuda T et al: Clustering of CMT1A duplication breakpoints in a $700 \mathrm{bp}$ interval of the CMT1A-REP repeat. Hum Mutat 1998; 11: 109-113.

24 Stronach EA, Clark C, Bell C et al: Novel PCR-based diagnostic tools for Charcot-Marie-Tooth type 1A and hereditary neuropathy with liability to pressure palsies. J Peripher Nerv Syst 1999; 4: $117-122$.

25 Navon R, Timmerman V, Lofgren A et al: Prenatal diagnosis of Charcot-Marie-Tooth disease type 1A (CMT1A) using molecular genetic techniques. Prenat Diagn 1995; 15: 633-640.

26 Timmerman V, Rautenstrauss B, Reiter LT et al: Detection of the CMT1A/HNPP recombination hotspot in unrelated patients of European descent. J Med Genet 1997; 34: 43-49.

27 Seeman P, Mazanec R, Zidar J et al: Charcot-Marie-Tooth disease type $1 \mathrm{~A}(\mathrm{CMT} 1 \mathrm{~A})$ and hereditary neuropathy with liability to pressure palsies (HNPP): reliable detection of the CMT1A duplication and HNPP deletion using 8 microsatellite markers in 2 multiplex PCRs. Int J Mol Med 2000; 6: 421-426.

28 Latour P, Boutrand L, Levy N et al: Polymorphic short tandem repeats for diagnosis of the Charcot-Marie- Tooth 1A duplication. Clin Chem 2001; 47: 829-837. 
29 Bieche I, Olivi M, Champeme MH et al: Novel approach to quantitative polymerase chain reaction using real-time detection: application to the detection of gene amplification in breast cancer. Int J Cancer 1998; 78: 661 -666.

30 Wang SC, Klein RD, Wahl WL et al: Tissue coexpression of LBP and CD14 mRNA in a mouse model of sepsis. J Surg Res 1998; 76: $67-73$.

31 Bassler HA, Flood SJ, Livak KJ et al: Use of a fluorogenic probe in a PCR-based assay for the detection of Listeria monocytogenes. Appl Environ Microbiol 1995; 61: 3724-3728.

32 Wilke K, Duman B, Horst J: Diagnosis of haploidy and triploidy based on measurement of gene copy number by real-time PCR. Hum Mutat 2000; 16: $431-436$
33 Aarskog NK, Vedeler CA: Real-time quantitative polymerase chain reaction. A new method that detects both the peripheral myelin protein 22 duplication in Charcot- Marie-Tooth type 1A disease and the peripheral myelin protein 22 deletion in hereditary neuropathy with liability to pressure palsies. Hum Genet 2000; 107: $494-498$.

34 Miller SA, Dykes DD, Polesky HF: A simple salting out procedure for extracting DNA from human nucleated cells. Nucleic Acids Res 1988; 16: 1215.

35 Heid CA, Stevens J, Livak KJ et al: Real time quantitative PCR. Genome Res 1996; 6: 986-994.

36 Lie YS, Petropoulos CJ: Advances in quantitative PCR technology: 5' nuclease assays. Curr Opin Biotechnol 1998; 9: 43-48. 